\title{
Hommage
}

\section{Henri Tardieu (1946-2010)}

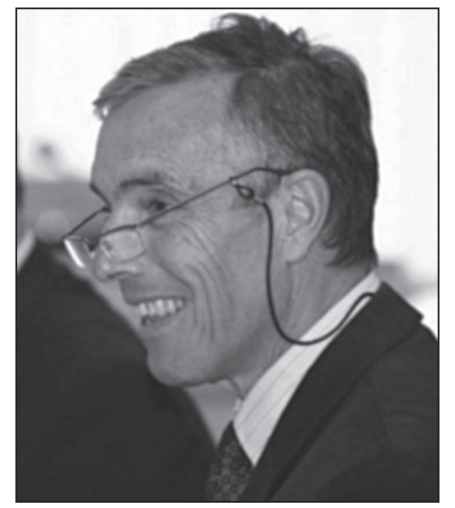

$\mathrm{H}$

enri Tardieu nous a quittés le 2 avril 2010.

Passionné par l'eau et l'environnement, il était devenu Ingénieur du génie rural, des eaux et des forêts après avoir été l'un des plus jeunes diplômés de l'École polytechnique.

Originaire de la région parisienne, il commence sa carrière professionnelle dans le développement agricole au Sénégal et en Algérie. En 1974, il entre à la Compagnie d'aménagement des Coteaux de Gascogne (CACG) dont il deviendra directeur général en 1990 et qu'il quittera à sa retraite en 2007. Tout au long de ces années, il a contribué à la définition de la majorité des schémas d'aménagement hydraulique de bassin en Adour-Garonne, participé au développement de la gestion intégrée des associations syndicales autorisées (ASA) d'irrigation, lancé les commissions de concertation par rivière, dont celle du système Neste, et contribué à la définition et à la mise en œuvre des pratiques modernes d'une gestion quantitative de l'eau. C'est également dans le cadre de son mandat de directeur général qu'il accompagne l'insertion régionale de la CACG dans les collectivités locales. Depuis 2007, Henri Tardieu demeurait très impliqué dans les réflexions conduites au sein de sa région en tant que membre du comité de bassin Adour-Garonne, et depuis peu membre du Conseil économique et social régional de Midi-Pyrénées.

Sa profonde connaissance de l'agriculture irriguée et de l'aménagement des territoires du Sud-Ouest de la France a permis à Henri Tardieu de développer une vision originale de la gestion de l'eau fondée sur le partage et la solidarité. Tout au long de sa carrière, il a su la faire partager en France et à l'international en tant que président de l'Association française pour l'eau, l'irrigation et le drainage (AFEID) et de vice-président de la Commission internationale des irrigations et du drainage (CIID).

Henri Tardieu avait à cœur que l'AFEID porte des messages forts sur l'eau et l'alimentation. Il a ainsi contribué à rapprocher l'AFEID de l'Association scientifique et technique pour l'eau et l'environnement, de la Société hydrotechnique de France et de l'Académie de l'eau, afin de développer des synergies pour leur assurer un plus grand rayonnement local, national, et international. Il participait activement à la définition de la position gouvernementale française lors des forums mondiaux de l'eau. Il soutenait aussi les initiatives de l'AFEID pour faire vivre le débat sur l'eau, l'agriculture et l'environnement dans les territoires français.

Au sein de la CIID, Henri Tardieu a été le maitre d'œuvre de la réorganisation des activités techniques. Il y a également animé les réflexions sur le thème de la stratégie de gestion de l'eau par bassin hydrographique et ce, jusqu'à la dernière réunion à New Delhi en décembre 2009. Il a présidé l'équipe chargée de préparer un rapport sur la durabilité socio-économique des services fournis par les projets d'irrigation, de drainage et de maitrise des crues, qui sert depuis plusieurs années de référence à la Banque mondiale. L'an dernier, il a contribué de manière remarquable à la prise de position de la CIID sur le thème "L'eau et la nourriture pour mettre fin à la pauvreté et la faim " au $5^{\mathrm{e}}$ Forum mondial de l'eau à Istanbul. 


\section{Hommage}

Henri Tardieu était convaincu de l'importance de l'enseignement et de la recherche pour penser et construire les nouveaux paradigmes d'un secteur qui dépasse la vision de l'ingénieur, et dont la maîtrise constitue l'une des clés de la satisfaction des besoins alimentaires de l'humanité.

Henri Tardieu, enfin, était proche des jeunes et aimait enseigner. Depuis les années 1990, il donnait régulièrement des conférences à AgroParisTech, sur les équilibres à trouver entre agriculture irriguée et environnement. La base de ses cours reposait sur son métier exercé à la CACG, mais aussi sur ses nombreuses expertises internationales, dans lesquelles il trouvait les ressorts pour illustrer son propos et provoquer les étudiants au débat. Mais s'il est un projet qui suscita particulièrement son enthousiasme, c'est la mise en place d'un séminaire annuel sur la gestion de l'eau, ouvert aux doctorants du Nord comme du Sud, sous l'égide de l'AFEID et de l'unité mixte de recherche (UMR) G-Eau. Il s'y impliqua chaque année, dans la revue des présentations, et dans la présidence des ateliers ; il apportait alors au doctorant le regard du professionnel et de l'employeur sur les travaux et leur utilité sociale. Il trouvait là un complément indispensable, expliquait-il, aux comités de thèse, puis aux jurys auxquels il participait régulièrement.

Son charisme, ses compétences, sa curiosité intellectuelle et sa bonne humeur étaient unanimement appréciés, ce dont témoignent les très nombreux messages de peine, d'amitié et d'admiration de celles et ceux qui l'ont connu dans tous les cercles auxquels il participait, français et internationaux.

Sami Bouarfa ${ }^{1}$ $<$ sami.bouarfa@cemagref.fr $>$ François Brelle $<$ francois.brelle@canal-de-provence.com>

Thierry Rieu ${ }^{3}$ $<$ rieu@agroparistech.fr> Jean-Philippe Torterotot ${ }^{4}$ $<$ jean-philippe.torterotot@cemagref.fr> Alain Vidal $<$ a.vidal@cgiar.org> Alain Villocel ${ }^{\sigma}$ $<$ a.villocel@cacg.fr>

${ }^{1}$ Cemagref/UMR G-Eau

${ }^{2}$ Société du Canal de Provence

${ }^{3}$ AgroParisTech

${ }^{4}$ Cemagref

${ }^{5}$ CGIAR-CPWF

${ }^{6}$ Compagnie d'aménagement des Coteaux de Gascogne 